\title{
What is the evidence on the inheritance of resistance alleles in populations of lepidopteran/ coleopteran maize pest species: a systematic map protocol
}

\author{
Achim Gathmann ${ }^{*}$ and Kai U Priesnitz
}

\begin{abstract}
Background: Lepidopteran and coleopteran species are the most important insect pests in maize. These pests can be controlled by the cultivation of genetically modified crops expressing insecticidal Bt-proteins. The long term success of this technology demands resistance management strategies to delay the evolution of resistance. The most efficient delay in resistance evolution can be expected if the inheritance of resistance is completely recessive. Therefore this information is important to predict the potential to evolve resistance of a pest/crop system.

Methods: Our review will take the form of an evidence map. Different databases will be used to collect as much data as possible to have a broad data basis to model and assess the potential for evolution of resistance of different crop-Bt-protein-species combinations. All identified articles will be recorded in a database. Relevant studies will be identified in a three step approach based on inclusion criteria. The systematic map output will be provided in a database. The database will be easily searchable and freely accessible. Additionally the data will be summarized in a narrative report.
\end{abstract}

Keywords: Systematic review, Bacillus thuringiensis, Bt-protein, Insect pests, Maize, Corn, Resistance, Inheritance

\section{Background}

Lepidopteran and coleopteran species are the most important pests in maize. The efficacy of common control measures is affected by several factors: The stalk boring behaviour of some pest species reduces exposure to insecticides, which makes it difficult to control these species effectively. The resistance evolution makes insecticides ineffective, the environmental harm associated with the use of insecticides should be reduced or economic considerations lead to higher pest pressure (no tillage, maize on maize cultivation). An alternative approach to common control measures is the use of genetically modified (GM) crops expressing Bt-proteins.

However, one concern with growing $B t$-maize is the potential for evolution of resistance. Resistance evolution against agricultural control measures is a well known

\footnotetext{
* Correspondence: achim.gathmann@bvl.bund.de

Federal Office of Consumer Protection and Food Safety, Department Genetic Engineering, Unit Coexistence and GMO-Monitoring, Mauerstr, 39-42, Berlin
} 10117, Germany

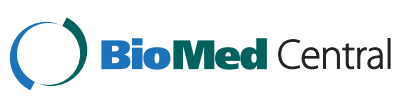

(c) 2014 Gathmann and Priesnitz; licensee BioMed Central Ltd. This is an Open Access article distributed under the terms of the Creative Commons Attribution License (http://creativecommons.org/licenses/by/4.0), which permits unrestricted use, distribution, and reproduction in any medium, provided the original work is properly credited. The Creative Commons Public Domain Dedication waiver (http://creativecommons.org/publicdomain/zero/1.0/) applies to the data made available in this article, unless otherwise stated. problem for more than 100 years [1]. It occurs regularly where pest populations are exposed to a uniform, strong and continuous selection pressure [1-3] and is therefore expected also for insect resistant GM crops expressing $B t$ proteins. In consequence $B t$-products might lose their effectiveness against pests, both as a conventional spray application and as a transgenic trait of GM crops. Therefore resistance management must accompany the cultivation of $B t$-crops to delay the evolution of resistance to B. thuringiensis products [4].

The regulatory risk assessment does not consider the occurrence of resistance evolution in general, but the efficacy of strategies suggested by applicants slowing down the resistance evolution. The most common strategy is the "high-dose/refuge" strategy (e.g. [1,5]). The common approach to forecast the evolution of resistance is the use of models. 
The principles of the high-dose/refuge strategy used in these models are that (1) the protein is killing nearly all pest organisms feeding on GM crops (high dose), (2) the frequency of resistance alleles is low, (3) the inheritance of resistance is fully recessive, (4) rare resistant pests surviving on $B t$-crops mate with abundant susceptible pests from nearby refuges of host plants without $B t$-proteins [5], and (5) fitness costs are associated with the evolution of resistance [6]. Therefore data on the biology of the target organisms, the characteristics of the modified plant and the GM trait are needed to support the model with all relevant information. In the case that not all requirements for the high dose refuge strategies are fulfilled, a modified strategy or additional measures might be needed.

Models showed that most efficient delay in resistance evolution can be expected if the inheritance of resistance is completely recessive [7]. Only in such cases heterozygous offspring resulting from crosses between resistant and susceptible individuals are expected to be susceptible to the $B t$-toxin [6].

Therefore this information is important to predict the potential to evolve resistance of a pest/crop system. This was confirmed by stakeholders who were consulted during a workshop and by a priorisation process of the review questions.

The goal of the evidence map will be to identify relevant information for modelling and assess the potential for evolution of resistance of different crop-Bt-proteinspecies combinations. Data will be based on independent data collections.

\section{Objective of the review}

One key assumption for the high-dose/refuge strategy is that the inheritance of resistance is fully recessive. Therefore data on the inheritance of resistance is needed to be sure about the recessiveness of the inheritance in the target organism. The availability of such data is essential to enable risk assessors and managers to assess which $B t$-maize events allow a successful resistance management regarding several target organisms. Another aim of the review is to identify potential knowledge gaps. Based on the above remarks, the following review question was formulated.

\section{What is the evidence on the inheritance of resistance alleles $(\mathrm{O})$ in populations of lepidopteran/coleopteran maize pest species $(P)$}

The research question has the following components:

$\mathrm{P}=$ Population:

The populations considered are all lepidopteran or coleopteran pest species in maize, which are intended to be controlled by Bt-maize in Europe. Additionally, lepidopteran or coleopteran pest species should be considered, which might be of economic relevance in maize cultivation. The selection of considered species is based on an extended review initiated by EFSA [8].

$\mathrm{O}=$ Outcome:

Data on inheritance of lepidopteran and coleopteran genes conferring resistance to $B t$-proteins will be extracted from the evaluated articles.

\section{Methods}

This review is part of the EU funded project GRACE to collate available evidence on evolution of resistance to $B t$-crops relevant in Europe. The review team and the stakeholder group for this review are formed by scientists from the project and representatives from NGOs, authorities, industry and academics.

\section{Search strategy}

The aim of the search is to obtain unbiased and comprehensive information relevant to the review question. Both published and unpublished data should be collated. Different sources of information will be searched in order to maximize the coverage of the search. All searches will be conducted in English.

\section{Search terms}

Combinations of the following search terms will be applied to the selected databases. * denotes a wild card for zero or more characters. The search terms are related to population and outcome. The search will include title, abstract and keywords. As all databases and websites vary in the way they handle complex search strings and the use of Boolean operators the exact search strings used will be listed in the appendix of the review.

Population: (lepidopter* OR butterfl* OR coleopter* OR

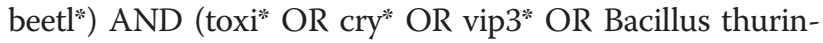
giensis* OR bt).

Outcome: (suscept* OR resistan* OR bioassay OR inheritance).

Several search strings have been tested in a preliminary search with Thomson Reuters (formerly ISI) Web of Science. The results are listed in Table 1. Search string number three will be chosen because of the most comprehensive search results.

\section{Data bases}

The following data bases will be considered:

- Thomson Reuters (formerly ISI) Web of Science, New York, USA, http://apps.webofknowledge.com

- Scopus by Elsevier, Amsterdam, The Netherlands, http://www.scopus.com/

- CAB abstracts, CABI, Wallingford, United Kingdom, http://www.cabdirect.org/ 
Table 1 Scoping results with different search strings (performed on 14.01.2013)

\begin{tabular}{|c|c|c|}
\hline $\mathrm{Nr}$ & Search string & Web of Science \\
\hline \multirow[t]{2}{*}{1} & $\begin{array}{l}\text { TS }=\left(\left(\text { lepidopter* OR butterfl* OR coleopter* OR beet }\left.\right|^{*}\right) \text { AND (europe) AND }\right. \\
(\text { (Bt Maize OR Bt corn OR transgenic maize OR transgenic corn) }\end{array}$ & 19 \\
\hline & $\begin{array}{l}\text { NOT (cotton OR rice OR soy*)) AND (Cry* OR Bt protein* OR Bt toxin OR Bacillus } \\
\text { thuringiensis toxin OR vip3*) AND (toxicity OR resistan* OR bioassay OR inheritance)) }\end{array}$ & \\
\hline \multirow[t]{2}{*}{2} & $\begin{array}{l}\left.\text { TS }=\left((\text { lepidopter* OR butterf })^{*} \text { OR coleopter* OR beet }\right)^{*}\right) \text { AND }((\text { Bt Maize OR Bt } \\
\text { corn OR transgenic maize OR transgenic corn) }\end{array}$ & 419 \\
\hline & $\begin{array}{l}\text { NOT (cotton OR rice OR soy*)) AND (Cry* OR Bt protein* OR Bt toxin OR Bacillus } \\
\text { thuringiensis toxin OR vip3*) AND (toxicity OR resistan* OR bioassay OR inheritance)) }\end{array}$ & \\
\hline 3 & $\begin{array}{l}\text { TS }=(\text { lepidopter* OR butterfl* OR coleopter* OR beetl*) AND TS }=\text { (toxi* OR cry* } \\
\text { OR vip3* OR Bacillus thuringiensis* OR bt) AND TS }=\text { (suscept* OR resist* OR inheritance) }\end{array}$ & 3838 \\
\hline
\end{tabular}

The asterisk $(*)$ denotes a wildcard for zero or more characters.

- Science Direct by Elsevier, Amsterdam, The Netherlands, http://www.sciencedirect.com/

- JSTOR by Ithaka, USA, http://www.jstor.org/

\section{Search engines}

Additionally the search will be supplemented by the use of internet meta search engines. For this Google Scholar (http://scholar.google.com) will be used. The search terms will be simplified and restricted to pdf or word files. The first 50 hits will be examined from this search.

\section{Specialist sources}

Additional grey literature or other useful data will be provided by the stakeholder group. Therefore known experts in the field of resistance management will be contacted and asked for unpublished data such as Diploma thesis, PhD thesis, technical reports or similar information. The scientists contacted and the information delivered will be recorded and listed in the report. Furthermore data collected in monitoring reports of the authorisation holders will be considered. Additionally applications for placing on the market will be screened for inheritance of resistance data. However these data can only considered upon approval by the consent holder.

\section{Study inclusion criteria}

All identified articles will be recorded in a database using the software Reference Manager Professional Version 12. Relevant studies will be identified in a three step approach based on inclusion criteria described below. In the first step articles will be excluded if their titles are considered irrelevant based on the inclusion criteria. In the second step articles will be excluded if their abstract is considered irrelevant. In both steps, a conservative approach will be used, meaning that if there is any doubt about the relevance of the article it will be retained. In the third step the full article will be viewed for the relevant studies.

The first and second step will be conducted independently by two reviewers. To avoid bias of reviewers a kappa test will be undertaken to guarantee consistency in the interpretation of the selection process. Therefore $10 \%$ of the papers (up to a maximum for 300 in the first step and 100 for the second step) will be checked and the level of agreement evaluated. If the kappa rating is below 0.6 , discrepancies in the decision making need to be discussed and adjusted to assess the remaining articles.

\section{Inclusion criteria}

\section{Relevant subject(s)/population(s)}

Pest species of maize with economic relevance or regionally economic relevance in Europe will be considered. The selection of species is based on EFSA data base listing arthropods species in crop fields as follows. Different names for the same species will be considered (in brackets) to minimize information loss by the existence of synonymies [9].

Lepidoptera: Ostrinia (Pyralis, Pyrausta) nubilalis, Sesamia nonagrioides, Agrotis segetum, Helicoverpa (Heliothis) armigera, Agrotis ipsilon (ypsilon), Autographa gamma, Mythimna (Pseudaletia) unipuncta, Acronicta rumicis, Mamestra brassicae, Sesamia cretica, Xylena vetusta

Coleoptera: Diabrotica virgifera virgifera.

\section{Relevant outcomes}

Studies which examine the inheritance of resistance genes

\section{Study quality assessment}

In the case of an evidence map no risk of bias assessment will be performed, but data are extracted which reflect the quality of the studies. For example, the number of replications and the number of test organisms will be recorded (Table 2). 
Table 2 List of possible variables extracted for the evidence map on the baseline susceptibility of insect pests to Bt-proteins

\begin{tabular}{|c|c|c|c|}
\hline Variable name & Definition & Type & Closed terms \\
\hline Article_id & Unique identification number assigned to each publication & integer & No \\
\hline Author & Author(s) of the listed publication. & text & No \\
\hline Publication_year & Year of publication of study & integer & No \\
\hline Citation & Citation, e.g. journal name, volume and page numbers & text & No \\
\hline Title & Title of the publication & text & No \\
\hline Was_peer_reviewed & Indicates whether study was published in a peer reviewed journal. & string & Yes \\
\hline Country & Country where insects were collected & string & Yes \\
\hline Expmt_num & $\begin{array}{l}\text { Number of experiment within a study (e.g. different species, } \\
\text { population, Bt-toxins, years, etc.) }\end{array}$ & string & No \\
\hline Bt_protein & Bt-protein used for the toxicity tests & string & Yes \\
\hline Target_order & Target taxonomic order & string & Yes \\
\hline Target_family & Target taxonomic family & string & Yes \\
\hline Target_genus & Target taxonomic genus & string & Yes \\
\hline Target_species & Target taxonomic species & string & Yes \\
\hline Test_method & $\begin{array}{l}\text { Used test methodology such as surface test, incorporation } \\
\text { test, test with discriminate dose or plant material }\end{array}$ & & \\
\hline Stage & Stage of the test organism & string & Yes \\
\hline No_test_organisms & Number of test organisms & real & No \\
\hline No_doses & Number of Bt protein doses used & real & No \\
\hline Control_no_test_organism & Number of test organism in the control & real & No \\
\hline Dose_no_test_organism & Number of test organism in the treatment & real & No \\
\hline Model_used & Statistical model used to calculate the inheritance of resistance & string & Yes \\
\hline h & $\begin{array}{l}\text { Effective dominance } h \text { at specific concentration } \\
\text { ( } h=0 \text { fully recessive, } h=1 \text { fully dominant) }\end{array}$ & string & Yes \\
\hline Tested_generations & No. of generations tested of the target organism & real & no \\
\hline Stability_resistance & Stability of resistance/generations & real & No \\
\hline Mode_of_inheritance & Description of the mode of inheritance & string & Yes \\
\hline
\end{tabular}

Given is the variable name in the database, the definition of the variable, the type, and whether the variable content is restricted to closed (predefined) terms.

\section{Data extraction strategy}

In addition to the authors of the studies, the date and the type of article will be recorded. Possible variables are the tested insect species, the country or region where the insect pests were collected, the description of the Cry protein used. The availability of data regarding the stability of the resistance and the inheritance of the resistance will be mapped.

A preliminary data extraction and coding sheet is annexed to the protocol. In the process two reviewers are involved. Reviewer one will extract the data. Results will be checked by a second reviewer.

\section{Data analysis}

The systematic map output will be provided in a database. The studies will be categorized using the variables presented in Table 2 . The database will be easily searchable and freely accessible. Additionally the data will be summarized in a narrative report. The presented information will be used as a starting point for additional focussed reviews but will be used additionally to identify important knowledge gaps.

\section{Competing interests \\ The authors declare that they have no competing interests.}

\section{Authors' contributions}

AG designed the review questions, wrote the review protocol and will conduct the literature search with subsequent data extraction and synthesis. KUP was involved in writing the review protocol and will conduct the literature search as well as the data extraction. Both authors read and approved the final manuscript.

\section{Acknowledgements}

The project was funded within EU-FP7 project: GMO Risk Assessment and Communication of Evidence (GRACE); Grant Agreement KBBE-2011-6-311957.

Received: 7 February 2014 Accepted: 29 May 2014

Published: 3 July 2014 


\section{References}

1. Andow DA: The risk of resistance evolution in insects to transgenic insecticidal crops. Coll Biosafety Rev 2008, 4:142-199.

2. Roush RT: Managing pests and their resistance to Bacillus thuringiensis: Can transgenics be better than sprays? Biocontro/ Sci Technol 1994, 4:501-516.

3. Tabashnik BE: Delaying insect adaption to transgenic plants: seed mixtures and refugia reconsidered. Proc Roy Soc B Biol Sci 1994, 255:7-12.

4. Hokkanen $\mathrm{HM}$, Wearing $\mathrm{CH}$ : The safe deployment of Bacillus thuringiensis genes in crop plants: conclusions and recommendations of OECD worhshop on ecological implications of transgenic crops containing $\mathrm{Bt}$ toxin genes. Biocontrol Sci Technol 1994, 4:399-404.

5. Tabashnik BE, Gould F: Delaying corn rootworm resistance to Bt corn. J Econ Entomol 2012, 105:767-776.

6. Devos Y, Meihls LN, Kiss J, Hibbard BE: Resistance evolution to the first generation of genetically modified Diabrotica-active Bt-maize events by western corn root worm: management and monitoring considerations. Transgenic Res 2013, 22:269-299.

7. Bates SL, Zhao J-Z, Roush RT, Shelton AM: Insect resistance management in GM crops: past, present and future. Nat Biotechnol 2005, 25:57-62.

8. Meissle M, Álvarez-Alfageme F, Malone LA, Romeis J: Establishing a database of bio-ecological information on non-target arthropod species to support the environmental risk assessment of genetically modified crops in the EU. In Supporting Publications 2012: EN-334. Parma, Italy: European Food Safety Authority (EFSA); 2014:170. http://www.efsa.europa. eu/en/publications.htm.

9. Encyclopedia of Life (EOL): In http://eol.org/.

doi:10.1186/2047-2382-3-13

Cite this article as: Gathmann and Priesnitz: What is the evidence on the inheritance of resistance alleles in populations of lepidopteran/coleopteran maize pest species: a systematic map protocol. Environmental Evidence 2014 3:13.

\section{Submit your next manuscript to BioMed Central and take full advantage of:}

- Convenient online submission

- Thorough peer review

- No space constraints or color figure charges

- Immediate publication on acceptance

- Inclusion in PubMed, CAS, Scopus and Google Scholar

- Research which is freely available for redistribution 